\title{
The Combined Use of the Wiener Polynomial and SVM for Material Classification Task in Medical Implants Production
}

\author{
Ivan Izonin \\ Lviv Polytechnic National University, PIT department, Lviv, 70913, Ukraine \\ E-mail: ivanizonin@gmail.com
}

\begin{abstract}
Andriy Trostianchyn, Zoia Duriagina, Roman Tkachenko, Tetiana Tepla, Nataliia Lotoshynska
Lviv Polytechnic National University, Lviv, 70913, Ukraine

E-mail: \{trostianchyn, roman.tkachenko\}@gmail.com, \{zduriagina, tetiana.l.tepla, natlot\}@ukr.net
\end{abstract}

Received: 16 June 2018; Accepted: 24 July 2018; Published: 08 September 2018

\begin{abstract}
This document presents two developed methods for solving the classification task of medical implant materials based on the compatible use of the Wiener Polynomial and SVM. The high accuracy of the proposed methodology for solving this task are experimentally confirmed. A comparison of the proposed methods with existing ones: Logistic Regression; Linear SVC; Random Forest; SVC (linear kernel); SVC (RBF kernel); Random Forest + Wiener Polynomial is carried out. The duration of training of all methods that described in work is investigated. The article presents the visualization of all method results for solving this task.
\end{abstract}

Index Terms-Machine learning, classification, medical implants, Wiener polynomial, SVM, titanium allows.

\section{INTRODUCTION}

The process of the creating new materials for aggressive environments at different temperatures imposes certain some requirements on their microstructure and properties. Prospective for this purpose is stainless steels, pure titanium, and alloys based on it. These materials are widely used in various industries, especially in the production of rocket and aircraft engineering [1].

Today, titanium and titanium alloys have been used in medicine [2]. This is due to their unique properties, in particular, biological inertia in relation to the human body, a high specific strength and a corrosion resistance in a variety of aggressive environments, including sterilizing substances, plasma, and blood [2]. Firstly, this contributes to the widespread use of titanium alloys for medical products, including implants, dentures, etc. Secondly, the systematization of numerous experimental studies and the results of clinical trials $[3,4]$ allows the creation of large databases containing the functional properties of these materials.

These factors create the preconditions for the artificial intelligence tools $[5,6]$ to be used for processing such databases for the synthesis of new materials or their operational properties identification from a huge set of available and investigated features. In the manufacture of products, it will allow making a decision quickly about choosing the optimal material's composition with predefined properties. As a result, it will be possible significantly to reduce financial resources and time in comparison with traditional investigation and design methods.

The current state of machine learning algorithms includes many similar methods [7] so their proper selection, adaptation or improvement is an important problem to solve this task.

The remainder of this paper is organized as follows. Section 2 describes related works and problem statement. Section 3 is devoted to the proposed methods. Section 4 presents an experimental research. Conclusions and future work are given in the final section.

\section{RELATED WorkS AND PROBLEM STATEMENT}

The problem of developing methods for the effective classification of titanium alloy powders is described in [8, 9]. In particular, in [8], a computational intelligence method for determining the optimal microstructure and titanium alloy powders properties is developed. The tool used for this is the Probabilistic Neural Network. The advantage of the method is to assess the probability of assigning the input sample to a particular class. This enables an expert to make timely and correct decisions about the use or non-use of a material for the elements design of the necessary equipment. However, it makes a subjective assessment in the development of the product, and in combination with the low classification's accuracy (about 70\%) imposes a number of restrictions on the use of the proposed classification tool.

In [9] the authors describe a method for the identification of titanium alloys powders based on the use 
of a Kolmogorov-Gabor polynomial (Wiener polynomial) and the Random Forest algorithm (RF). The results of the task solution due to the use of this method have been significantly improved (more than 95\% accuracy), however, the selection and setting of optimal parameters of the method do not provide a complete solution to the task. In addition, increase the number of the RF's trees to achieve a higher classification accuracy results causes to increase the time delays during its work. This situation is complicated in the case of Big Data or data streams processing [10, 11].

The aim of this work is to develop methods of the titanium alloys classification for the medical implants production. Such methods should, firstly, to provide high accuracy for designing products. Inappropriately identifiable material properties during its classification may cause defects during operation and reduce the expiration date [12]. Consequences and health damage in the case of a malfunction, breakage or complete failure of medical products are not to be estimated. Secondly, the developed methods should provide high speed in the training mode.

The advantage of using machine learning techniques to solve this task lies in the fact that they allow combining various factors (characteristics of titanium alloy powders) that effect on the properties of the materials which made from such alloys. This, in turn, increases the sensitivity of such methods to rather rare combinations of factors, which can provide a more accurate classification.

The main argument of using SVM as a tool for effectively solving a given task is the ability of this tool to work with a much smaller data sample compared to, for example, neural networks $[13,14]$.

\section{PROPOSED METHODS}

Support Vector Machine (SVM) is one of the most widely used methodologies for data classification in Material Science [15, 16]. The linear SVM classifier proposed by V.N. Vapnik in 1953 gained its development in the early 1990's by creating a non-linear classifier using arbitrary kernel functions.

The basic idea of the method is as follows. It is necessary to find such a separating line between points on the plane, which will provide the most accurate procedure for assigning points to the corresponding classes (below and above the line). Such a line, according to the idea of this method, should be constructed as far away as possible from the points nearest to it from the two classes. This operation principle provides a more reliable classification [17]. When the spaces of high dimensions are considered, instead of the line, we consider the hyperplane, which will divide the classes of given objects. Detailed mathematical descriptions of the method are given in [18].

Among the main benefits of using this toolkit in the Material Science field are:

- the method provides uniqueness of the solution;

- the method is characterized by very high speed;
- the method provides the possibility of processing large amounts of information;

- the method provides some versatility by the possibility of using both existing kernels of functions and offering their own;

- there are developed optimal schemes of memory allocation and computational procedures for SVM realization to improve the efficiency of the method.

However, SVM has a number of disadvantages, the most important of which for solving the classification task is the low accuracy for noisy input data.

The multiparametric dependences of each data vector (Fig. 1), which are characteristic of this task, sometimes lead to an ineffective solution of the task, in particular by tools of computing intelligence [19]. In [8], the solution accuracy of the classification task (on the same data as in the work) using the Probabilistic Neural Network was $70 \%$.

Incorrect identification of the material class, in this case, can lead to a number of defects in medical implants created on its basis. This can lead to both large material losses and a threat to human life (in the case of the presence of defective planes in vital organs). The need to increase the results accuracy of the materials classification task, cause to the use of effective tools for solving this task. Wiener polynomial (WP), as a universal approximator, can increase the solution accuracy of this task [9]:

$$
\begin{aligned}
& Y\left(x_{1}, \ldots, x_{n}\right)=a_{i}+\sum_{i=1}^{n} a_{i} x_{i}+\sum_{i=1}^{n} \sum_{j=i}^{n} a_{i, j} x_{i} x_{j}+ \\
& +\sum_{i=1}^{n} \sum_{j=i}^{n} \sum_{l=j}^{n} a_{i, j, l} x_{i} x_{j} x_{l}+\ldots \\
& \ldots+\sum_{i=1}^{n} \sum_{j=i}^{n} \sum_{l=j}^{n} \ldots \sum_{z=k-1}^{n} a_{i, j, l, \ldots, \mathrm{z}} x_{i} x_{j} x_{l} \ldots x_{z}
\end{aligned}
$$

where $k$ is a polynomial degree, $x_{1}, \ldots, x_{n}$ is the variables.

This is justified by the Weierstrass first theorem [9]. However, procedures for finding the coefficients of this polynomial by existing methods [20], especially in cases of large dimensional data, are not always effective.

That is why the paper proposes a method for data classification based on the combined use of Wiener Polynomial and SVM. This will ensure sufficient speed of the classification method by using SVM, as well as increase the accuracy of the solution to this task by using the Wiener Polynomial.

The algorithmic realization of the developed method involves the following basic steps:

1. The input attributes (20 characteristics) consider as members of the Wiener polynomial (excluding zero member);

2. Classification of a vector based on the search using SVM of a Wiener polynomial's zero-member and coefficients for non-zero members. 
The performance of the proposed method will depend on the implementation of step 2. As you know, SVM results largely depend on the choice of both the kernel and the value of the regularization parameter for the geometric difference [18].

That is why, for the investigation of the classification materials method for the search of the Wiener polynomial coefficients (step 2), two SVM implementations from two different Python libraries: LIBSVM [21] and LIBLINEAR [22] were used. These libraries contain similar SVM-based classifiers: C-Support Vector Classification (SVC) in LIBLINEAR (with the different kernels) and Linear Support Vector Classification (Linear $S V C)$, which, in fact, is another implementation of the $S V C$ classifier with the linear kernel. Detailed mathematical descriptions of the $S V C$ method are given in [23], and the Linear SVC method in [24].

The main distinguishing feature of both implementations is a different set of parameters that are used to solve the classification task. The parameters of both methods are given in Table 1 of Appendix A.

The combined use of Linear SVC and Wiener polynomials let's called Method 1, and the use of SVC (with the different kernel) and Wiener polynomial let's call Method 2.

\section{EXPERIMENTAL RESULTS}

For modeling of the proposed methods, it was used the DataSet from [8, 9]. Based on the studies from [8, 25], it was possible to distinguish four groups of characteristics that effect a composition of an alloy, which can be synthesized from four different titanium alloys powder components (Fig. 1).

In our case, every group contained from three to six characteristics, which in sum gave 20 independent attributes that were attributes of each input vector.

In [9], 480 observations were conducted. Each of them has one of four alloy characteristics according to the required properties (Table 1). Such an alloy are based on four different materials IMI 125, Ti-Al-Mo-Zr, Ti-6Al$4 \mathrm{~V}$, Ti-Al-V-Zr. Thus, Data Set of 480 observations, each containing 20 inputs and one output, that describing the class of the resulting alloy (Table 1), was constructed.

To simulate the described methods, the dataset was randomly divided into the test and training samples in accordance with 20 and $80 \%$. A numerical representation of the representatives of each class for both the test and the training samples are showed in Table 1.

Table 1. Numerical representation of classes of the material in test and training samples

\begin{tabular}{|c|c|c|c|}
\hline $\begin{array}{c}\text { Number } \\
\text { of class }\end{array}$ & Characteristics of the material & $\begin{array}{c}\text { Quantity in } \\
\text { the test } \\
\text { sample }\end{array}$ & $\begin{array}{c}\text { Quantity in } \\
\text { the training } \\
\text { sample }\end{array}$ \\
\hline 1 & Excellent material properties & 32 & 150 \\
\hline 2 & Optimal material properties & 24 & 94 \\
\hline 3 & Material with possible defects & 32 & 100 \\
\hline 4 & Defective material & 8 & 40 \\
\hline
\end{tabular}

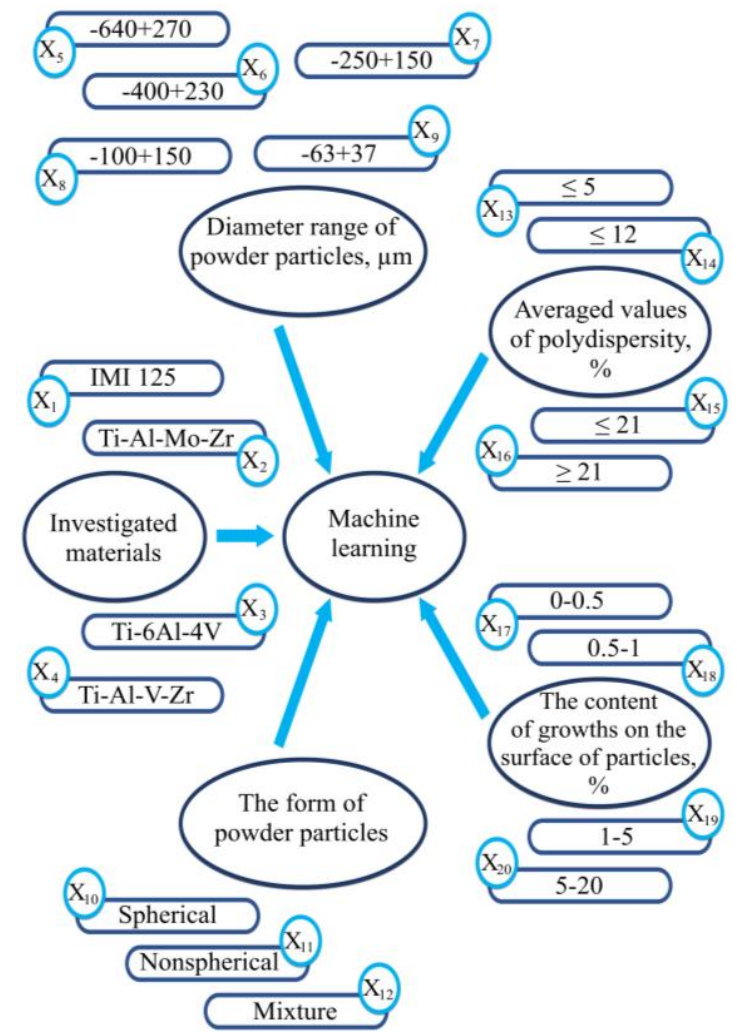

Fig.1. Elements of Data Set for modelling

The Method 1 and the Method 2 are implemented in Python using libraries LIBSVM [21] and LIBLINEAR [22]. Visualization of the results of the used methods was conducted using the Orange Software [26].

The classification results were evaluated according to the formula:

$$
\begin{gathered}
\begin{array}{c}
\text { The number of } \\
\text { Accuracy }=\frac{\text { correctly classified vectors }}{\text { The test sample size }} \\
\text { The number of }
\end{array} \\
=\frac{\text { correctly classified vectors }}{96}
\end{gathered}
$$

Table 2, based on (1), presents the results of comparing both developed methods with existing ones. As can be seen from Table 2, the best results for solving the classification task by both developed methods were shown.

Table 2. Comparison of the accuracy for different methods

\begin{tabular}{|c|c|c|}
\hline$\#$ & Method & Accuracy \\
\hline 1 & Logistic Regression & 63,54 \\
\hline 2 & SVC (RBF kernel) & 63,54 \\
\hline 3 & Linear SVC & 63,54 \\
\hline 4 & SVC (linear kernel) & 76,04 \\
\hline 5 & Random Forest & 93,75 \\
\hline 6 & Method from [9] & 95,83 \\
\hline $\mathbf{7}$ & Method 2 & $\mathbf{9 5 , 8 3}$ \\
\hline $\mathbf{8}$ & Method 1 & $\mathbf{9 6 , 8 8}$ \\
\hline
\end{tabular}


Fig. 2 presents the results of comparing the accuracy of the work of all methods in training and testing modes. As can be seen from the graphs, the developed methods show a slight difference in the values of accuracy in both modes of operation. This testifies to the possibility of practical application of these methods for solving applied tasks of Material Science.

Fig. 4 shows scatter plots of all investigated methods. As can be seen from Fig.4 (g, h, i) the best results are shown by the methods $6,7,8$ from Table 2 . It should be noted that besides the low accuracy of the work, methods 1, 2, 3 and 4 of Table 2 are identified samples of class 4 as representatives of class 1 or 2 (Fig.4 (b, c, e, f)).

This increases the likelihood of the creation of a medical implant part from a material that is generally not recommended for use (a class 4 from Table 1). The risk of such a situation leads to a number of negative consequences, and such methods are not recommended for use in solving the task.

An important point when applying machine learning methods is the time of their work. In addition to ensuring high accuracy, similar methods should provide a high speed in training mode

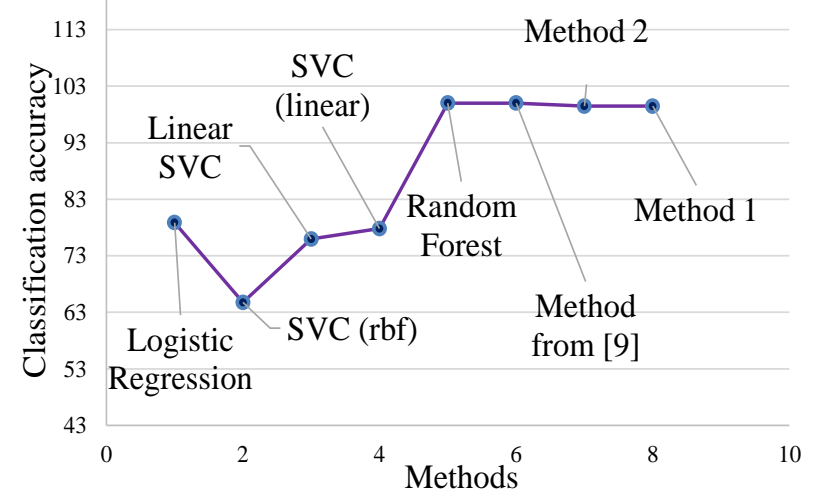

a)

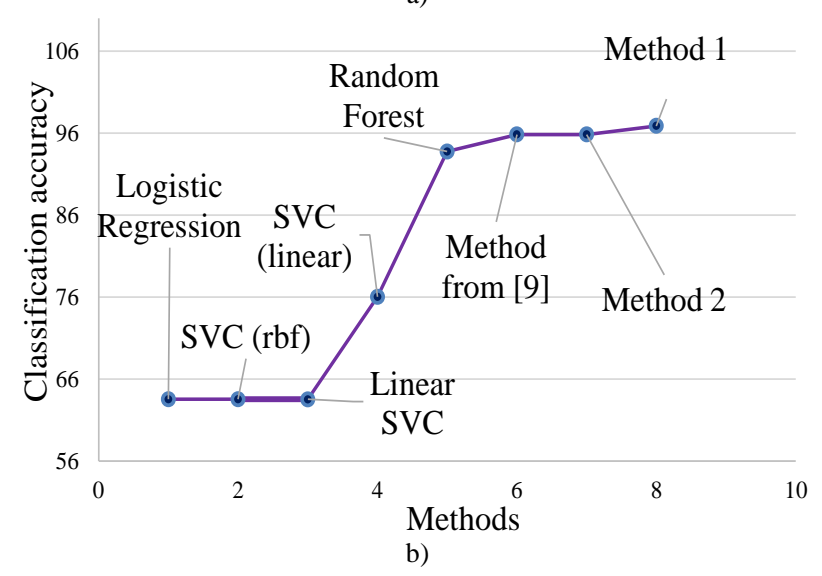

Fig.2. Visual comparison of the classification accuracy for the developed methods with the existing ones: a) in training mode; b) in test mode.

In this paper, the investigated of the comparing the results of the training time for all methods was conducted. In Fig. 3 the results of the conducted experiments are presented. As can be seen from Fig. 3, the Method 1
(Linear SVC + Wiener Polynomial)) shows a very small training time. However, compared with the base Linear $\mathrm{SVC}$, the proposed method is working is 1.7 times slower. This is due to the implementation of step 1 of the method. It involves the representation of input data in the form of members of the Wiener polynomial, which substantially increases the dimension of each input vector. That is why the length of the training procedures are increasing significantly.

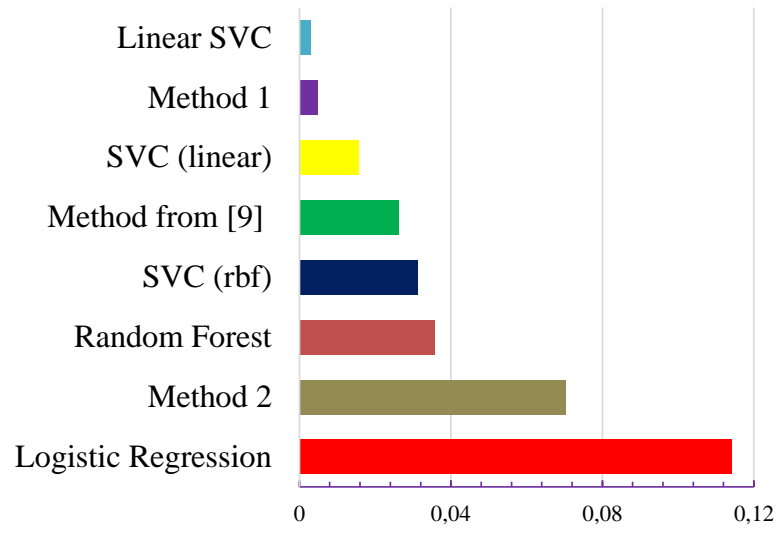

Fig.3. Comparison of training time of the developed machine leaning methods with existing ones.

Nevertheless, comparing the developed method with the method of [9] shows an increase in speed more than 7 times with the same values in accuracy. Regarding the Method 2 (SVC (linear kernel) + Wiener Polynomial). It increases the training time compared to the basic SVC (linear kernel).

However, the advantage of the proposed method is the high accuracy of the solution for the classification task, which is $32.29 \%$ higher than the accuracy of the basic method. In addition, the Method 2 is worse (the classification accuracy) only compared to the Method 1 from all investigation methods in this paper.

Based on the obtained results (the accuracy and speed) of the proposed methods, it can be asserted that:

- the developed methods show a high accuracy of the task of identifying the material class, which determines the possibility of their practical use for solving such tasks;

- the Method 2 should be used for solving classification tasks in the Material Science field in case that doesn't impose restrictions for their training time;

- the Method 1 shows the greatest accuracy of the solution of the classification task among all the considered ones. It only shows a slightly worse result compared to the basic method for the performance of the training procedure;

- based on the accuracy and speed of the Method 1 work, it can be used to solve applied classification tasks in the case of large dimensions of the input data;

- the proposed approach shows a high accuracy of calculating the Wiener polynomial coefficients, 
which enables its application in the fields of medicine[27], education [28], image processing
[29], in particular, for solving tasks of both classification and regression.

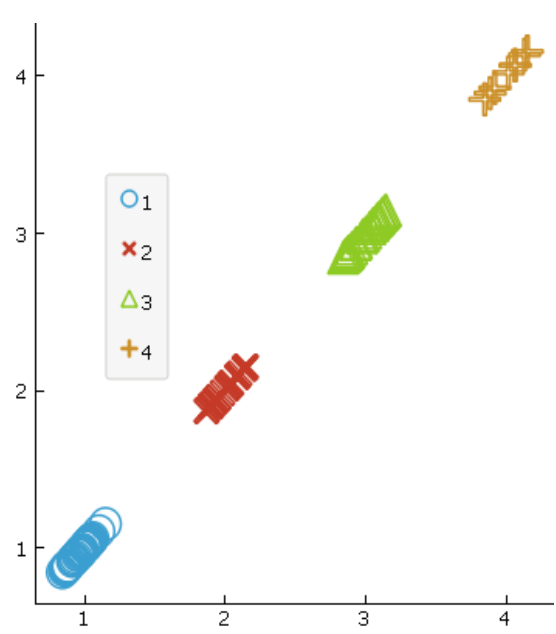

a)

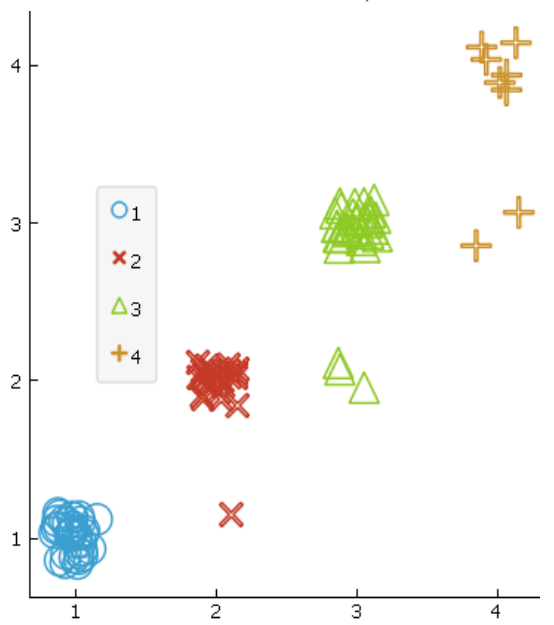

d)

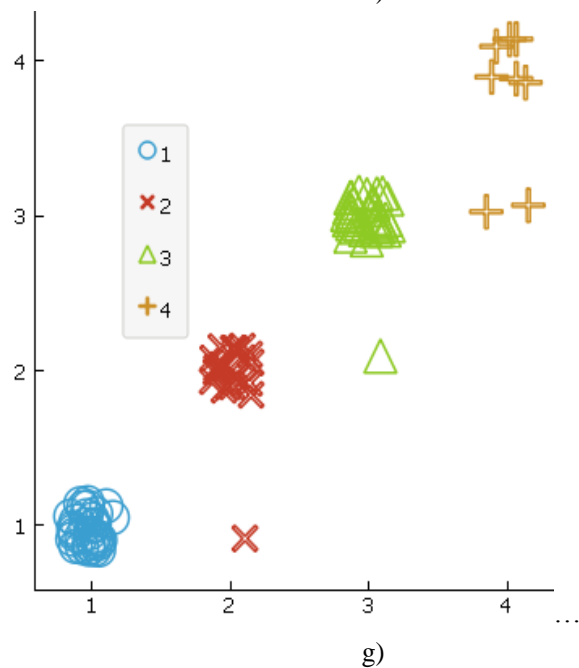

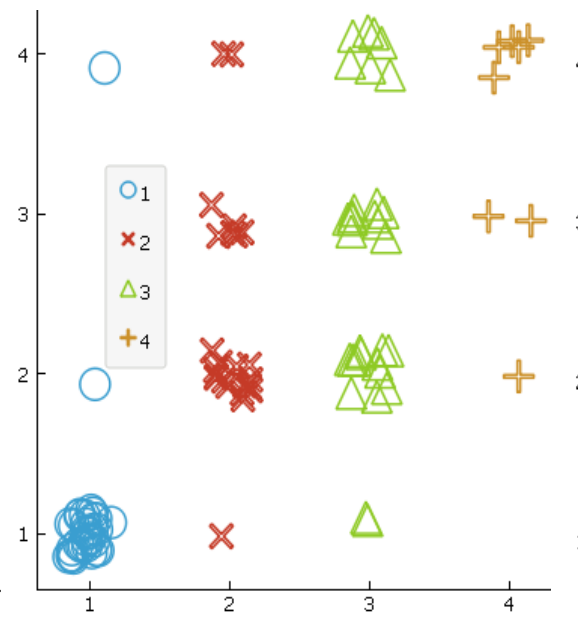

b)
ᄂ

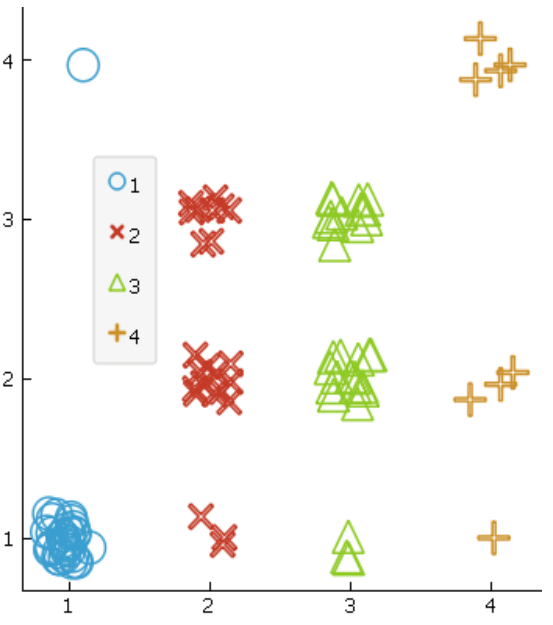

c)

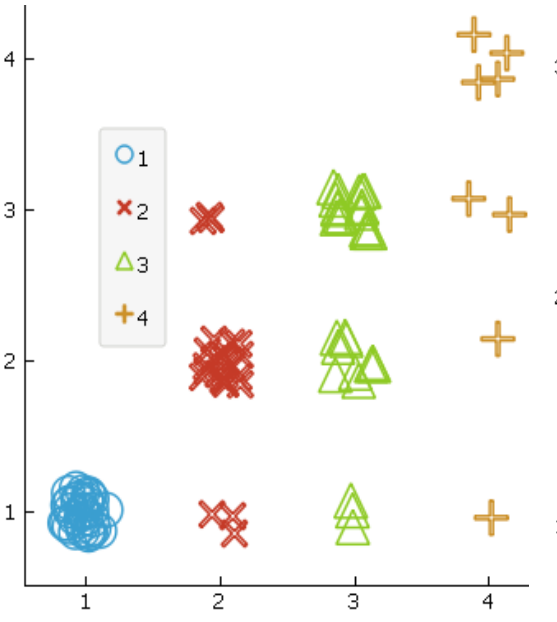

e)

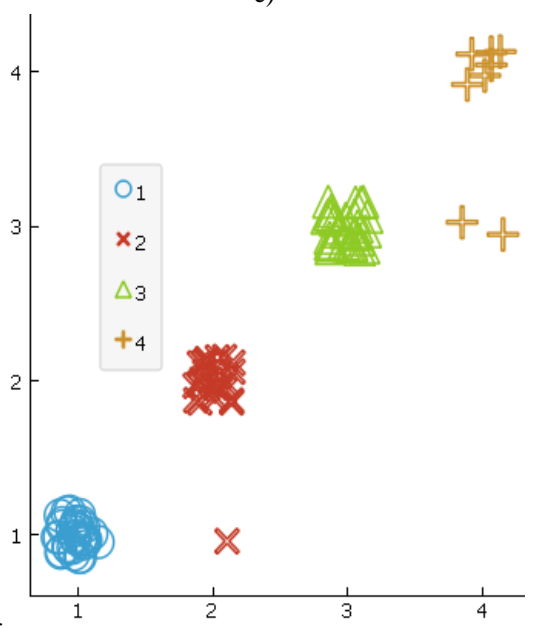

h)

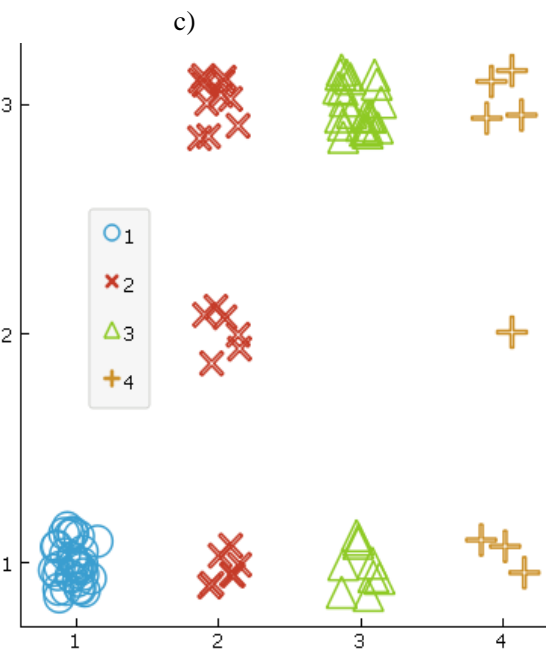

f)

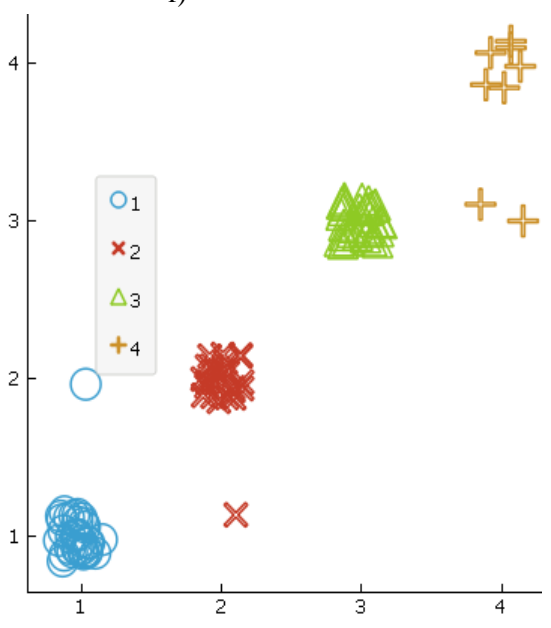

i)

Fig.4. Visual comparison of the developed methods with existing ones. On the x-axis there are true values, on the y-axis there are obtained values by one of the methods: a) Initial status (ideal variant); b) Logistic Regression; c) Linear SVC; d) Random Forest; e) SVC (linear); f) SVC (rbf); g) Method from [9] (Random Forest + Wiener Polynomial); h) Linear SVC + Wiener Polynomial ; i) SVC (linear) + Wiener Polynomial. 


\section{APPENDIX A PARAMETERS OF THE METHODS}

The basic parameters of the developed methods and the methods that were used for comparison can be seen in Table $3[18,30]$.

Table 3. Basic parameters of the methods

\begin{tabular}{|c|c|c|}
\hline \# & Method & Parameters \\
\hline 1 & Logistic Regression & $\begin{array}{l}\text { multi_class : 'multinomial'; } \\
\text { class_weight : 'balanced'; } \\
\text { solver : 'lbfgs'. }\end{array}$ \\
\hline 2 & $\begin{array}{l}\text { SVC with RBF kernel } \\
\text { (C-Support Vector } \\
\text { Classification) }\end{array}$ & $\begin{array}{c}\text { coef0 : } 0.0 ; \\
\text { shrinking : True; } \\
\text { C : } 0.3 ; \\
\text { kernel : 'rbf'; } \\
\text { degree : } 3 ; \\
\text { gamma : 'auto'; } \\
\text { class_weight : None; } \\
\text { verbose : False; } \\
\text { max_iter : -1; } \\
\text { probability : False; } \\
\text { tol : } 0.001 ; \\
\text { cache_size : } 200 ; \\
\text { decision_function_shape : 'ovr'; } \\
\text { random_state : None }\end{array}$ \\
\hline 3 & $\begin{array}{l}\text { Linear SVC (Linear } \\
\text { Support Vector } \\
\text { Classification) }\end{array}$ & $\begin{array}{c}\text { tol : } 0.0001 ; \mathrm{C}: 1.0 ; \\
\text { multi_class : 'ovr'; } \\
\text { penalty : '12'; } \\
\text { loss : 'squared_hinge'; } \\
\text { dual : True; } \\
\text { verbose : } 0 ; \\
\text { random_state : None; } \\
\text { max_iter : } 10 ; \\
\text { fit_intercept : True; } \\
\text { intercept_scaling : } 1 ; \\
\text { class_weight : None. }\end{array}$ \\
\hline 4 & $\begin{array}{c}\text { SVC with linear } \\
\text { kernel (C-Support } \\
\text { Vector Classification) }\end{array}$ & $\begin{array}{l}\text { kernel : 'linear'; } \\
\text { other parameters are the same as in } \\
\text { method } 2\end{array}$ \\
\hline 5 & Random Forest & $\begin{array}{c}\text { min_samples_split : } 2 \\
\text { n_estimators }: 10 \\
\text { random_state }: 0 \\
\text { max_depth }: \text { None. }\end{array}$ \\
\hline 6 & Method from [5] & $\begin{array}{l}\text { Wiener polynomial : second degree; } \\
\text { n_estimators : } 9 \text {; } \\
\text { other parameters are the same as in } \\
\text { method } 5\end{array}$ \\
\hline 7 & $\begin{array}{c}\text { Linear SVC + Wiener } \\
\text { Polynomial }\end{array}$ & $\begin{array}{l}\text { Wiener polynomial : second degree; } \\
\text { other parameters are the same as in } \\
\text { method } 3\end{array}$ \\
\hline 8 & $\begin{array}{l}\text { SVC (linear) + Wiener } \\
\text { Polynomial }\end{array}$ & $\begin{array}{l}\text { Wiener polynomial : second degree; } \\
\text { other parameters are the same as in } \\
\text { method } 4\end{array}$ \\
\hline
\end{tabular}

\section{CONCLUSIONS}

The paper describes two developed methods for the titanium alloys classification for medical purposes. They are based on combining of the Wiener polynomial and SVM. To implement the machine learning procedures, two similar implementations of the SVM method from different Python libraries were used.

The experimentally established effectiveness of the developed methods that provide the highest classification accuracy was compared with existing methods.The SVM's training time was evaluated experimentally. The Method 1 (Linear SVC + Wiener Polynomial) has been found to provide a high speed and the highest accuracy.
That is why it can be used for solving applied classification tasks in case of the necessity of taking into account the functional properties of structural materials. These properties, in turn, depend on the chemical composition and microstructure of the choosen materials, which determines the large dimensions of the input data (Big Data processing).

It has been found that the Method 2 (SVC (linear kernel) + Wiener Polynomial) should be used for solving classification tasks in cases that do not impose restrictions for the training time.

\section{ACKNOWLEDGMENT}

The authors would like to thank to Dr. Ihor Lemishka and Dr. Volodymyr Kulyk and senior researcher Anatolii Kovalchuk for the assistance with data preparation and participation in the development of Artificial Intelligence methods for solving applied tasks of Material Science.

\section{REFERENCES}

[1] O.P. Ostash, V.V. Kulyk, T.M. Lenkovskiy, Z.A. Duriagina, V.V. Vira and T.L. Tepla, "Relationships between the fatigue crack growth resistance characteristics of a steel and the tread surface damage of railway wheel", Archives of Materials Science and Engineering, vol. 90(2), pp. 49-55, 2018.

[2] A. T. Sidambe, "Biocompatibility of Advanced Manufactured Titanium Implants-A Review", Materials (Basel), vol. 7(12), pp. 8168-8188, 2014.

[3] N. Boyko, T. Sviridova, and N. Shakhovska, "Use of machine learning in the forecast of clinical consequences of cancer diseases", In 2018 7th Mediterranean Conference on Embedded Computing (MECO). IEEE, pp.43-56, June 2018.

[4] N. Melnykova, N. Shakhovska and T. Sviridova, "The personalized approach in a medical decentralized diagnostic and treatment," 2017 14th International Conference The Experience of Designing and Application of CAD Systems in Microelectronics (CADSM), Lviv, pp. 295-297, 2017. doi: 10.1109/CADSM.2017.7916139.

[5] Zh. Hu, Ye.V. Bodyanskiy and O.K. Tyshchenko, "A deep cascade neural network based on extended neo-fuzzy neurons and its adaptive learning algorithm," 2017 IEEE First Ukraine Conference on Electrical and Computer Engineering (UKRCON), Kiev, pp. 801-805, 2017. doi: 10.1109/UKRCON.2017.8100357

[6] Zh. Hu, Ye.V. Bodyanskiy and O.K. Tyshchenko, "A hybrid growing ENFN-based neuro-fuzzy system and its rapid deep learning," 2017 12th International Scientific and Technical Conference on Computer Sciences and Information Technologies (CSIT), Lviv, pp. 514-519, 2017. doi: 10.1109/STC-CSIT.2017.8098840.

[7] A. Kazarian, V. Teslyuk, I. Tsmots and M. Mashevska, "Units and structure of automated "smart" house control system using machine learning algorithms," 2017 14th International Conference The Experience of Designing and Application of CAD Systems in Microelectronics (CADSM), Lviv, pp. 364-366б 2017.

[8] Z.A. Duriagina, R.O. Tkachenko, A.M. Trostianchyn, I.A. Lemishka, A.M. Kovalchuk, V.V. Kulyk and T M. Kovbasyuk, "Determination of the best microstructure and titanium alloy powders properties using neural network", Journal of Achievements in Materials and Manufacturing 
Engineering, vol. 87 (1), pp. 25-31, 2018. doi: $10.5604 / 01.3001 .0012 .0736$

[9] R. Tkachenko, Z. Duriagina, I. Lemishka, I. Izonin and A. Trostianchyn, "Development of machine learning method of titanium alloys properties identification in additive technologies", EasternEuropean Journal of Enterprise Technologies, vol. 3, iss. 12 (93), 2018, pp. 23-31. DOI: 10.15587/1729-4061.2018.134319

[10] Zh. Hu, Ye.V. Bodyanskiy, O.K. Tyshchenko and O.O. Boiko ${ }_{2}$ "A Neuro-Fuzzy Kohonen Network for Data Stream Possibilistic Clustering and Its Online SelfLearning Procedure", Applied Soft Computing, vol. 68, pp.710-718, 2018.

[11] Ye.V. Bodyanskiy, O.K. Tyshchenko and D.S. Kopaliani, "An Evolving Connectionist System for Data Stream Fuzzy Clustering and Its Online Learning", Neurocomputing, vol. 262, pp. 41-56, 2017.

[12] Z. Duriagina, R. Holyaka, T. Tepla, V. Kulyk, P. Arras and E. Eyngorn, "Identification $\mathrm{Fe} 3 \mathrm{O} 4$ nanoparticles biomedical purpose by magnetometric methods", Biomaterials in Regenerative Medicine, Edited by Leszek A. Dobrzański, Publisher: InTech, P. 448, 2018, Chapter 17, pp. 379-407.

[13] Ye. Bodyanskiy, O. Vynokurova, G. Setlak, D. Peleshko and P. Mulesa, "Adaptive multivariate hybrid neuro-fuzzy system and its on-board fast learning", Neurocomputing, vol. 230, pp. 409-416, 2017. doi.org/10.1016/j.neucom.2016.12.042

[14] Ye. Bodyanskiy, O. Vynokurova, I. Pliss, D. Peleshko, and Yu. Rashkevych, "Hybrid generalized additive wavelet-neuro-fuzzy-system and its adaptive learning", In: Zamojski W., Mazurkiewicz J., Sugier J., Walkowiak T., Kacprzyk J. (eds) Dependability Engineering and Complex Systems. DepCoS-RELCOMEX 2016. Advances in Intelligent Systems and Computing, vol 470, pp 51-61, Springer, Cham, 2016. doi.org/10.1007/978-3-319-39639$2 \_5$

[15] K. Rajan "Materials informatics", Materials Today, vol. 8, iss. 10 , pp. $38-45,2005$. doi: 10.1016/s13697021(05)71123-8

[16] P. Raccuglia, K. C. Elbert, et. al. "Machine-learningassisted materials discovery using failed experiments", Nature, vol. 533, iss, 7601, pp. 73-76, 2016. doi: 10.1038 /nature 17439

[17] K. V. Vorontsov "Lectures on the Support Vectors Method", 2007, (in Russian). URL: www.ccas.ru/voron/download/SVM.pdf

[18] Support Vector Machines. URL: http://scikitlearn.org/stable/modules/svm.html

[19] V. Lytvyn, V. Vysotska, I. Peleshchak, I. Rishnyak and R. Peleshchak, "Time Dependence of the Output Signal Morphology for Nonlinear Oscillator Neuron Based on Van der Pol Model", International Journal of Intelligent Systems and Applications(IJISA), vol. 10, no. 4, pp. 8-17, 2018. DOI: 10.5815/ijisa.2018.04.02

[20] I. Dronyuk, M. Nazarkevych and O. Fedevych, "Synthesis of Noise-Like Signal Based on Ateb-Functions", In: Vishnevsky V., Kozyrev D. (eds) Distributed Computer and Communication Networks. DCCN 2015. Communications in Computer and Information Science, Springer, Cham, vol. 601, 2016.

[21] C.-C. Chang and C.-J. Lin, "LIBSVM: a library for support vector machines", ACM Transactions on Intelligent Systems and Technology, 2:27:1--27:27, 2011. Software available at http://www.csie.ntu.edu.tw/ cjlin/libsvm

[22] R.-E. Fan, K.-W. Chang, C.-J. Hsieh, X.-R. Wang and C.-
J. Lin. "LIBLINEAR: A library for large linear classification" Journal of Machine Learning Research, vol. 9, pp. 1871-1874, 2008.

[23] A.J. Smola and B. Schölkopf "A Tutorial on Support Vector Regression", Statistics and Computing archive, vol. 14, iss.3, p. 199-222, 2004.

[24] R.-E. Fan, P.-H. Chen and C.-J. Lin. "Working set selection using second order information for training SVM", Journal of Machine Learning Research, vol. 6, pp. 1889-1918, 2005.

[25] Z.A. Duriagina, T.L. Tepla and V.V. Kulyk "Evaluation of differences between $\mathrm{Fe} 3 \mathrm{O} 4$ micro- and nanoparticles properties", Acta Physica Polonica A, vol. 133(4), pp. 869-872, 2018.

[26] Data Mining Fruitful and Fun. URL: https://orange.biolab.si

[27] M.N.F. Fajila, M.A.C. Akmal Jahan, "The Effect of Evolutionary Algorithm in Gene Subset Selection for Cancer Classification", International Journal of Modern Education and Computer Science(IJMECS), Vol.10, No.7, pp. 60-66, 2018.DOI: 10.5815/ijmecs.2018.07.06

[28] Mukesh Kumar, A.J. Singh, Disha Handa,"Literature Survey on Student's Performance Prediction in Education using Data Mining Techniques", International Journal of Education and Management Engineering(IJEME), Vol.7, No.6, pp.40-49, 2017.DOI: 10.5815/ijeme.2017.06.05

[29] Suchit Purohit, Savita R. Gandhi," Application of Sparse Coded SIFT Features for Classification of Plant Images", International Journal of Image, Graphics and Signal Processing(IJIGSP), Vol.9, No.10, pp. 50-59, 2017.DOI: 10.5815/ijigsp.2017.10.06

[30] Scikit-learn Machine Learning in Python. URL: http://scikit-learn.org/stable/

\section{Authors' Profiles}

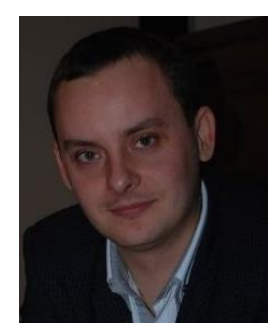

Ivan Izonin, graduated from Lviv Polytechnic National University in 2011 (MSc in Computer Sciences) and Ivan Franko National University of Lviv in 2012 (MSc in Economic Cybernetics). He got his Ph.D. in Artificial Intelligence in 2016. He is currently working as an Assistant at Publishing Information Technologies Department, Lviv Polytechnic National University, Lviv, Ukraine.

He has published more than 80 publications, including 8 patents for inventions, 1 tutorial. His major research interests are Computational Intelligence; High-Speed Neural-Like Systems; Non-iterative Machine Learning Algorithms.

Dr. Izonin participating in the developing two international Erazmus+ projects - MASTIS and DocHub. He is a Technical Committee Member of several international conferences.

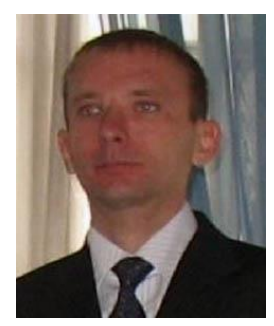

Andriy Trostianchyn graduated from Lviv Polytechnic National University in 1999 (MSc in Material Sciences). He got his Ph.D. in Material Sciences in 2005 in Karpenko physico-mechanical institute of the National Academy of Sciences of Ukraine. He is currently working as an Assistant in Department of Applied Materials Science and Materials Engineering, at Lviv Polytechnic National University, Lviv, 
Ukraine.

He has published more than 100 publications in national and international journals. His major research interests are Computational Intelligence and 3D printing technology for the production of aerospace equipment.

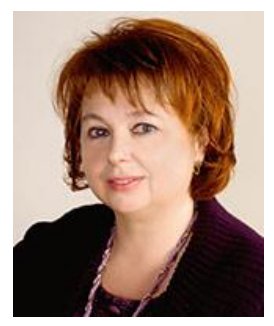

Zoia Duriagina. Prof., Doctor of Technical Sciences, Head of the Department «Applied Materials Science and Materials Engineering», Lviv Polytechnic National University, Ukraine. Prof., dr. hab. of the Lublin Catholic University, Poland.

She has published more than 218 scientific publications, including 5 scientific monographs (2 of them in English), 4 textbooks and 16 patents.

Scientific and research interest - Stainless steels and functional alloys. Surface engineering: application of functional (dielectric, resistive, heat-resistant, protective) coatings. Creating new thermoelectric materials. Investigation the properties of Ti- powders alloys: development of 3D printing technology for the production of aerospace equipment. Biomedical, medical materials and functional coatings.

Professor Duriagina has a several awards: the «Golden Medal of Polish Scientific Society of Materials Science», Gliwice, 2016-2017. Diploma of International expert in the field of materials science, Weihai, China, 2017. Member of the Academy of Higher Education of Ukraine (since 2008). Member of the World Academy of Materials and Manufacturing Engineering (since 2015).

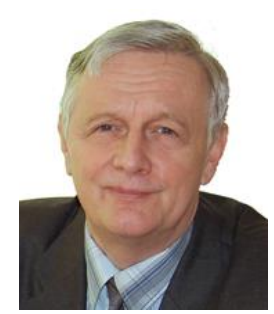

Roman Tkachenko graduated from Lviv Polytechnic National University in 1972. He took his Ph.D. degree in 1984 and Dr. Sc. degree in 2000. The topic of his Dr. Sc. thesis was "Neural networks of direct propagation with non-iterative training". $\mathrm{He}$ is currently working as a Head of the Publishing Information Technologies Department at Lviv Polytechnic National University, Lviv, Ukraine.

He has published more than 200 publications, including 12 patents for inventions, 3 tutorial. He is a developer of a new neural paradigm based on the Geometric Transformations Model, author of scientific research of high-speed intelligence technologies. His major research interests are neural and neuralfuzzy artificial intelligence means for Big Data tasks.

Professor Tkachenko is head of the scientific and technical projects in Ukraine and abroad on the development of artificial intelligence means based on the neural-like and neural-fuzzy structures.

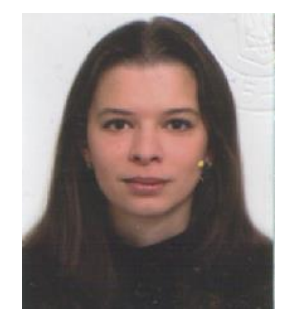

Tetiana Tepla, graduated from Lviv Polytechnic National University in 2001 and obtained MSc in material sciences. She got his Ph.D. in Material Science in 2008. She is currently working as Senior Lecturer in Department of Applied Materials Science and Materials Engineering, at Lviv Polytechnic National University, Lviv, Ukraine.

She has published more than 50 publications. Her major research interests are: steel and alloys with special properties; corrosion and corrosion protection; biocompatible materials.

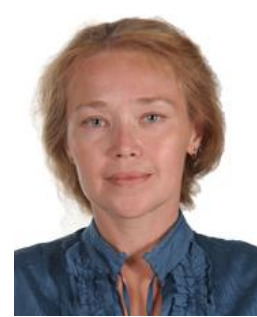

Nataliia Lotoshynska graduated from Ukrainian Academy of Printing in 1992. She took her Ph.D. degree in 1998. Currently she is working as a lecturer of the Publishing Information Technologies Department at Lviv Polytechnic National University, Lviv, Ukraine.

She has published more than 80 publications, including 4 patents for inventions, 2 textbooks. Scientific research in the field of printing technologies and software environment for production and accounting of paint mixes, in the field of printing technologies and materials of flat offset printing and 3D printing technologies.

How to cite this paper: Ivan Izonin, Andriy Trostianchyn, Zoia Duriagina, Roman Tkachenko, Tetiana Tepla, Nataliia Lotoshynska, "The Combined Use of the Wiener Polynomial and SVM for Material Classification Task in Medical Implants Production", International Journal of Intelligent Systems and Applications(IJISA), Vol.10, No.9, pp.40-47, 2018. DOI: 10.5815/ijisa.2018.09.05 